\title{
Short communication: Investigation of Coxiella burnetii occurrence in dairy sheep flocks by bulk-tank milk analysis and antibody level determination
}

\author{
A. L. García-Pérez, ${ }^{1}$ I. Astobiza, J. F. Barandika, R. Atxaerandio, A. Hurtado, and R. A. Juste \\ NEIKER-Instituto Vasco de Investigación y Desarrollo Agrario, Department of Animal Health, Berreaga 1, 48160 Derio, Bizkaia, Spain
}

\begin{abstract}
To estimate the prevalence of Coxiella burnetii in the dairy sheep population from the Basque Country (northern Spain), a study was carried out combining molecular and serological techniques. First, bulk-tank milk samples from 154 flocks belonging to the Latxa Breed Farmers Association were analyzed by PCR, with $22 \%$ of flocks testing positive for C. burnetii. Then, a selection of 34 flocks (7 PCR positive and 17 negative) was investigated for the presence of serum antibodies by ELISA test on 1,011 ewes (approximately 30 ewes per flock). A total of $8.9 \%$ of the animals were seropositive, $67.6 \%$ of the flocks had at least one seropositive animal, but only in $14.7 \%$ of them was seroprevalence greater than $25 \%$. Older ewes showed a significantly greater prevalence $(17.5 \%)$ compared with yearlings (7.5\%) or replacement lambs (1.5\%). A marginally significant association was found between seroprevalence and PCR detection of $C$. burnetii in bulk-tank milk. The widespread distribution of $C$. burnetii in the region advocates for the implementation of $\mathrm{Q}$ fever control strategies and highlights the potential risk of sheep as a reservoir and infection source for other domestic and wildlife species and the human population.
\end{abstract}

Key words: Coxiella burnetii, dairy sheep, bulk-tank milk, polymerase chain reaction and enzyme-linked immunosorbent assay

Q fever is endemic in northern Spain, where the largest series of Q fever pneumonia in the world has been reported (Maurin and Raoult, 1999). Cattle, sheep, and goats are considered the primary sources of infection for humans. Clinical signs of Coxiella burnetii infection are abortion in sheep and goats and reproductive failure in cattle. The uterus and mammary glands are the primary sites of infection in the chronic phase of $C$. burnetii in ruminants. Shedding of this pathogen into the environment occurs mainly during parturition via birth products, but shedding through milk and feces has also

Received September 1, 2008.

Accepted December 3, 2008.

${ }^{1}$ Corresponding author: agarcia@neiker.net been documented in sheep, cattle, and goats (Berri et al., 2001; Guatteo et al., 2006; Rodolakis et al., 2007). After lambing, ewes shed C. burnetii for a variable period of days, and several authors reported even longer bacterial excretion in fecal samples (Rodolakis et al., 2007). Thus, the formation of aerosols contaminated with infected lambing products is the most common infection source for humans, although consumption of contaminated milk products has been linked with seroconversion (Fishbein and Raoult, 1992).

Analysis of bulk-tank milk (BTM) samples is a relatively inexpensive approach for detection and prevalence estimation of different pathogens in dairy flocks. Coxiella burnetii-specific PCR (Willems et al., 1994; Berri et al., 2000) applied to these BTM samples can provide data on herd exposure to $C$. burnetii and infection prevalence estimations (Kim et al., 2005).

There are approximately 322,000 sheep in the Basque Country, of which $90 \%$ are Latxa dairy sheep. Only $12 \%$ of the 4,000 Latxa flocks have 100 or more sheep, and most flocks are managed by full-time sheep farmers. Latxa flocks lamb once a year with an early peak between November and February for ewes on their second lactation and higher and a second peak in March and April for first-lactation replacement ewe-lambs. Most lambs are weaned and sold 5 wk after lambing, and ewes are then milked for an average of 5 mo. Latxa sheep are commonly housed in winter and during milking, and many flocks have access to summer communal mountain pastures. A retrospective PCR study recently carried out in sheep flocks with abortions in this region showed a prevalence of $C$. burnetii-positive flocks of around 9\% (Oporto et al., 2006), revealing the importance of sheep as a reservoir of this zoonotic pathogen. Therefore, a more representative study was carried out focused on detecting the presence of $C$. burnetii DNA in BTM from dairy sheep flocks in the Basque Country and evaluating its correspondence with Q fever seroprevalence. To this end, taking advantage of a parallel study on border disease in BTM, all the flocks belonging to the Latxa Breed Farmers Association (154 flocks) were visited in March and April 2005, when mainly ewes in second and higher lactations were being milked. The study flocks represented $32 \%$ of the farms with a 
minimum of 100 sheep. A short questionnaire was completed to compile information regarding the size of the flock, number of lactating ewes, and abortion history. One hundred milliliters of BTM per farm was collected into sterile plastic tubes, and DNA was extracted from somatic cells using QIAgen Mini Kit (Qiagen Iberia S.L., Madrid, Spain) according to the manufacturer's instructions, including a negative control (PBS) for every batch of 5 samples. Polymerase chain reaction amplification using primers targeting a transposon-like repetitive region of the $C$. burnetii genome was performed as described by others (Willems et al., 1994; Berri et al., 2000), with 150 ng of extracted DNA being added to each reaction. The proportion of flocks with a BTM positive result for DNA of C. burnetii was $22 \%$ (Table 1). Even though DNA extraction directly from somatic cells is not expected to give the best results for Coxiella (Rodolakis et al., 2007), PCR identified a large number of farms with $C$. burnetii shedders. Although the presence of DNA alone does not demonstrate an active infection or shedding of viable organisms, these results suggested that $C$. burnetii was present in the sheep population. Logistic regression models to analyze possible associations between flock size and PCR status did not show any significant association.

A fraction of flocks $(22 \%, 34 / 154)$ were selected for a seroprevalence study and visited in autumn and winter of 2005/2006, before lambing started. These flocks were a convenience sample obtained from a study on border disease virus infection in which flocks were selected according to their bulk milk ELISA result. Blood samples taken in each flock from the jugular vein from a maximum of 30 ewes (10 replacement lambs $>6$ mo, 10 ewes 1 to 2 yr old, 10 ewes $>2$ yr) were used to $\operatorname{detect} C$. burnetii antibodies using a commercial indirect ELISA (ELISA Cox kit, Laboratoire Service International, Lissieu, France). An index of the tested serum optical density to the difference between positive and negative controls was calculated. Serum samples with indices $\leq 40$ were considered negative, whereas samples with indices $>40$ were considered positive for $C$. burnetii antibodies.
The antibody ELISA had been validated for individual serum samples from experimentally infected animals by the manufacturer using PCR as the reference test to determine sensitivity (Se) and specificity $(\mathbf{S p})$, yielding estimates of 100\% Se and 95\% Sp. Positive ELISA results (90 of 1,011) set the overall individual apparent prevalence at $8.9 \pm 1.8 \%$ (95\% confidence interval, CI). Taking into consideration the test Se and Sp estimates according to the Rogan-Gladen and Youden corrections (Greiner and Gardner, 2000), the true prevalence estimate at the $95 \%$ CI would be $4.1 \pm 1.8 \%$. The proportions of seropositive animals across age categories were analyzed using Fisher exact test, and older ewes ( $>2$ yr old) showed a significantly greater prevalence $(17.5 \% ; P<0.05)$ compared with yearlings $(7.5 \%)$ and replacement ( $>6$ mo old) lambs (1.5\%; Table 1$)$. Flocks were considered positive when at least one animal had a positive reaction against $C$. burnetii. Thus, $67.6 \%$ of the flocks $(23 / 34)$ were positive, but prevalences $>25 \%$ were observed in only 5 flocks $(14.7 \% ; 5 / 34)$. The distribution of seroprevalence in positive flocks and the proportion within each age group are shown in Figure 1. No significant association was found between border disease and $C$. burnetii seroprevalences as shown by Chi square test analysis, thus ruling out any biased results due to sample selection. Still, the flock antibody prevalence observed in this study was higher than in other seroprevalence studies carried out in sheep in other regions of Spain (reviewed by Pascual-Velasco, 1996). The ELISA test used herein was based on an antigen isolated from ruminants (strain recovered from an ovine placenta at Institut National de la Recherché Agronomique, Tours, France, from an abortion of sheep), which offers better sensitivity than ELISA tests that use Nine Mile antigen obtained from ticks (Kovacova et al., 1998; Rodolakis, 2006; Rodolakis et al., 2007). In a previous study carried out in the same region years ago, the complement fixation test showed a flock prevalence of $40 \%$ and an individual seroprevalence of $6.4 \%$ (Sáez de Ocáriz et al., 1987). The higher prevalence observed in the present study is probably

Table 1. Polymerase chain reaction (bulk tank milk) and ELISA (sera) results

\begin{tabular}{lcccc}
\hline & & \multicolumn{2}{c}{ PCR/ELISA positive } & \\
\cline { 3 - 4 } Sample & $\mathrm{n}$ & $\mathrm{n}$ & $\%$ & \multirow{2}{c}{$95 \% \mathrm{CI}^{1}$} \\
\hline Bulk-tank milk & 154 & 34 & 22.1 & \pm 6.5 \\
Sera & & & & \\
Flocks & 34 & 23 & 67.6 & \pm 15.7 \\
Sheep & 1,011 & 90 & 8.9 & \pm 1.8 \\
$>2$ yr & 343 & 60 & 17.5 & \pm 4.0 \\
1 to 2 yr old & 334 & 25 & 7.5 & \pm 2.8 \\
$<1$ yr old & 334 & 5 & 1.5 & \pm 1.3 \\
\hline
\end{tabular}

${ }^{1} \mathrm{CI}=$ confidence interval. 


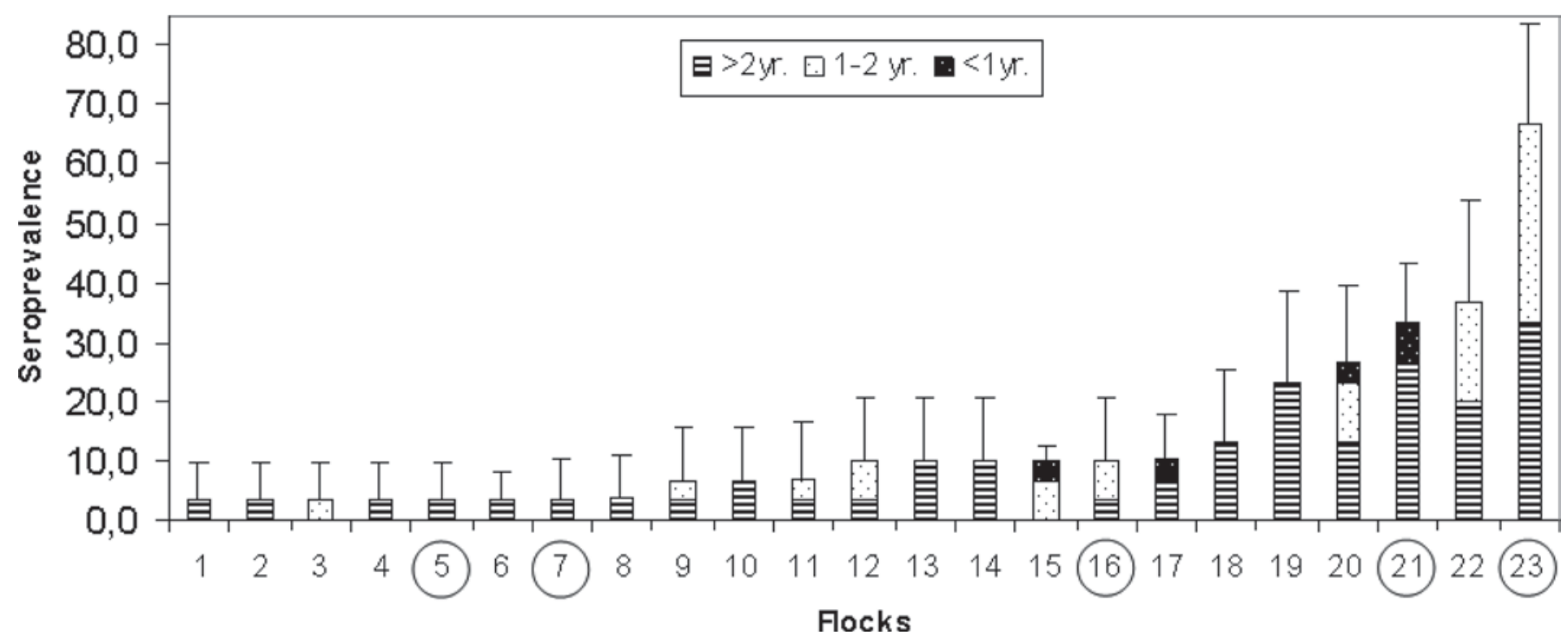

Figure 1. Frequency distribution of seroprevalence among the 23 flocks considered positive. The columns are divided to show the proportion within each age group. Whiskers show the upper $95 \%$ confidence interval for each flock. Flock numbers with PCR-positive bulk-tank milk are circled.

due to the greater sensitivity of ELISA compared with the complement fixation test (Kovacova et al., 1998; Rousset et al., 2007), meaning that, in fact, the disease would not have expanded significantly in the last 20 yr. One remarkable observation is that only $14.7 \%$ of the studied flocks in the current had a seroprevalence $>25 \%$. Taking this into account and the results obtained by PCR in BTM (22\% of PCR positive flocks), it can be hypothesized that $C$. burnetii is widespread in the sheep flocks in this region. This high prevalence is in accordance with the high incidence of the disease in humans in the same region (Montes et al., 2006). Because no data are available regarding the importance of other species such as cattle or goats in the cycle of $C$. burnetii infection in this region, at present we can only confirm that sheep might be an important reservoir of this bacterium in this area.

Flocks that had a previous history of abortion showed a higher seroprevalence against Coxiella $(14.2 \%)$ than those that did not report abortion problems $(6.0 \%, P$ $>0.05)$. However, it was not possible to establish the real causes of abortion in affected flocks. Odds ratios for previous history of abortion related to seroprevalence were calculated using the logistic procedure in the SAS statistical package (SAS Institute, Cary, NC) in which several models based on different groupings of flock prevalence rates were tested. Logistic regression indicated that the group of flocks with a seroprevalence $>3 \%$ had an odds ratio of 8 for abortion history compared with those having $\leq 3 \%$ seroprevalence $(95 \%$ CI: 1.522-42.041). The association found herein between higher seropositivity and abortion has also been observed elsewhere in cattle (Cabassi et al., 2005) and sheep (Cetinkaya et al., 2000). However, flocks without abortions at sampling but with seropositive animals could have been infected in the past. In addition, there are reports in which infection with $C$. burnetii in cattle did not result in abortion in infected animals (Paiba et al., 1999), suggesting that infection can occur unnoticed.

The association between seroprevalence and detection of $C$. burnetii DNA in BTM in the flocks where both analyses were performed (34) was marginally significant by Fisher exact test $(P=0.10)$. Flocks with $\geq 30 \%$ seroprevalence $(3 / 34)$ had $66.7 \%$ frequency of PCR positive results, whereas those with $<30 \%$ seroprevalence had $16.1 \%$ positive results. Several flocks with a low number of seropositive animals had BTM PCR-positive results (Figure 1) indicating a recent introduction of the agent in the farm or a lack of seroconversion in several infected sheep (Berri et al., 2002; Rodolakis et al., 2007), although lack of sensitivity of the ELISA test cannot be ruled out. In any case, the time lag ( $6 \mathrm{mo}$ ) between PCR and serology tests may explain temporal changes in the status of flocks, particularly because of culling of nonproductive ewes after the end of lactation in summer months, which probably reduced seroprevalence.

Finally, the different seroprevalence levels observed among age groups indicated that replacement ewes (6 mo to 1 yr old) were not exposed to the bacteria until the lambing season, when increased excretion of the agent causes greater exposure rates (Maurin and 
Raoult, 1999). Most of the serum samples were collected between October and December 2005, before lambing took place, explaining the low level of reactors among young animals. In addition, replacement lambs are reared in a separate group in several flocks, joining the adult flock just before parturition. On the contrary, older ewes presented higher seropositivity, as antibodies remain in C. burnetii infection for months (Berri et al., 2001, 2002).

In summary, infection by $C$. burnetii in dairy sheep flocks in the Basque Country is widely spread and might represent a risk for the human population. At present, control measures in livestock based on vaccination using phase I vaccine in naturally infected flocks are being evaluated. This vaccine is not commercially available in Spain but it has been specially authorized by the Spanish Drug Agency for a follow-up study on flocks with infection confirmed by the combination of serological and PCR techniques; the first results will be available in a few months and reported elsewhere. Vaccination protects against abortion and reduces bacteria shedding through feces, milk, or vaginal exudates in experimentally infected animals (Arricau-Bouvery et al., 2005); currently, the effects of vaccination have only been evaluated in bovine commercial herds (Guatteo et al., 2008), not in commercial sheep flocks.

\section{ACKNOWLEDGMENTS}

This work was supported by funding from INIA (FAU2006-00002-C04-01), FEDER and the Department of Agriculture, Fisheries and Food of the Basque Government. We thank Inés Povedano (Department of Animal Health, NEIKER, Derio, Bizkaia, Spain) for laboratory assistance. We acknowledge the collaboration of veterinarians and farmers from the Latxa Breed Farmers Association.

\section{REFERENCES}

Arricau-Bouvery, N., A. Souriau, C. Bodier, P. Dufour, E. Rousset, and A. Rodolakis. 2005. Effect of vaccination with phase I and phase II Coxiella burnetii vaccines in pregnant goats. Vaccine 23:4392-4402.

Berri, M., K. Laroucau, and A. Rodolakis. 2000. The detection of Coxiella burnetii from ovine genital swabs, milk and fecal samples by the use of a single touchdown polymerase chain reaction. Vet. Microbiol. 72:285-293.

Berri, M., A. Souriau, M. Crosby, D. Crochet, P. Lechopier, and A. Rodolakis. 2001. Relationships between the shedding of Coxiella burnetii, clinical signs and serological responses of 34 sheep. Vet. Rec. 148:502-505.
Berri, M., A. Souriau, M. Crosby, and A. Rodolakis. 2002. Shedding of Coxiella burnetii in ewes in two pregnancies following an episode of Coxiella abortion in a sheep flock. Vet. Microbiol. 85:55-60.

Cabassi, C. S., S. Taddei, G. Donofrio, F. Ghidini, C. Piancastelli, C. F. Flammini, and S. Cavirani. 2006. Association between Coxiella burnetii seropositivity and abortion in dairy cattle of Northern Italy. New Microbiol. 29:211-214.

Cetinkaya, B., H. Kalender, H. B. Ertas, A. Muz, N. Arslan, H. Ongor, and M. Gurcay. 2000. Seroprevalence of coxiellosis in cattle, sheep and people in the east of Turkey. Vet. Rec. 146:131-136.

Fishbein, D. B., and D. Raoult. 1992. A cluster of Coxiella burnetii infections associated with exposure to vaccinated goats and their unpasteurized dairy products. Am. J. Trop. Med. Hyg. 47:3540.

Greiner, M., and I. A. Gardner. 2000. Application of diagnostic tests in veterinary epidemiologic studies. Prev. Vet. Med. 45:43-59.

Guatteo, R., F. Beaudeau, M. Berri, A. Rodolakis, A. Joly, and H. Seegers. 2006. Shedding routes of Coxiella burnetii in dairy cows: Implications for detection and control. Vet. Res. 37:827-833.

Guatteo, R., H. Seegers, A. Joly, and F. Beaudeau. 2008. Prevention of Coxiella burnetii shedding in infected dairy herds using a phase I C. burnetii inactivated vaccine. Vaccine 26:4320-4328.

Kim, S. G., E. H. Kim, C. J. Lafferty, and E. Dubovi. 2005. Coxiella burnetii in bulk tank milk samples, United States. Emerg. Infect. Dis. 11:619-621.

Kovacova, E., J. Kazar, and D. Spanelova. 1998. Suitability of various Coxiella burnetii antigen preparations for detection of serum antibodies by various tests. Acta Virol. 42:365-368.

Maurin, M., and D. Raoult. 1999. Q fever. Clin. Microbiol. Rev. $12: 518-553$.

Montes, M., G. Cilla, D. Vicente, D. Nieto, M. Ercibengoa, and E. Perez-Trallero. 2006. Gipuzkoa, Basque Country, Spain (19842004): A hyperendemic area of Q fever. Hospital Donostia. Ann. N.Y. Acad. Sci. 1078:129-132.

Oporto, B., J. F. Barandika, A. Hurtado, G. Aduriz, B. Moreno, and A. L. Garcia-Perez. 2006. Incidence of ovine abortion by Coxiella burnetii in northern Spain. Ann. N. Y. Acad. Sci. 1078:498-501.

Paiba, G. A., G. Lloyd, K. Bewley, G. J. Webster, L. E. Green, and K. L. Morgan. 1999. An investigaton of Coxiella burnetii infection at calving within a herd of dairy cows in England using PCR. Pages 387-392 in Rickettsiae and Rickettsia Diseases at the Turn of the Third Millennium. D. Raoult and P. Brouqui, ed. Elsevier, Paris, France.

Pascual-Velasco, F. 1996. Fiebre Q. Consejería Sanidad y Bienestar Social. Ed. Junta de Castilla y León, Zamora, Spain.

Rodolakis, A. 2006. Q fever, state of art: Epidemiology, diagnosis and prophylaxis. Small Rumin. Res. 62:121-124.

Rodolakis, A., M. Berri, C. Héchard, C. Caudron, A. Souriau, C. C. Bodier, B. Blanchard, P. Camuset, P. Devillechaise, J. C. Natorp, J. P. Vadet, and N. Arricau-Bouvery. 2007. Comparison of Coxiella burnetii shedding in milk of dairy bovine, caprine, and ovine herds. J. Dairy Sci. 90:5352-5360.

Rousset, E., B. Durand, M. Berri, P. Dufour, M. Prigent, P. Russo, T. Delcroix, A. Touratier, A. Rodolakis, and M. Aubert. 2007. Comparative diagnostic potential of three serological tests for abortive Q fever in goat herds. Vet. Microbiol. 124:286-297.

Sáez de Ocáriz, C., J. L. Gelabert, and R. A. Juste. 1987. Encuesta serológica sobre la difusión de algunas enfermedades del ganado ovino Latxo. Informes Técnicos no. 5. Servicio de Publicaciones del Dpto. de Agricultura y Pesca del Gobierno Vasco, VitoriaGasteiz, Spain.

Willems, H., D. Thiele, R. Frolich-Ritter, and H. Krauss. 1994. Detection of Coxiella burnetii in cow's milk using the polymerase chain reaction (PCR). Zentralbl. Veterinarmed. B. 41:580-587. 